\title{
Isolated Structures in Two-Dimensional Optical Superlattice
}

\author{
Xinhao Zou, Baoguo Yang, Xia Xu, Pengju Tang and Xiaoji Zhou ${ }^{1, *}$
}

(Dated: September 10, 2018)

\begin{abstract}
Overlaying commensurate optical lattices with various configurations called superlattices can lead to exotic lattice topologies and, in turn, a discovery of novel physics. In this study, by overlapping the maxima of lattices, a new isolated structure is created, while the interference of minima can generate various "sublattice" patterns. Three different kinds of primitive lattices are used to demonstrate isolated square, triangular, and hexagonal "sublattice" structures in a twodimensional optical superlattice, the patterns of which can be manipulated dynamically by tuning the polarization, frequency, and intensity of laser beams. In addition, we propose the method of altering the relative phase to adjust the tunneling amplitudes in "sublattices." Our configurations provide unique opportunities to study particle entanglement in "lattices" formed by intersecting wells and to implement special quantum logic gates in exotic lattice geometries.
\end{abstract}




\section{INTRODUCTION}

The system of ultracold atoms trapped in optical lattices is a versatile tool to simulate systems in condensed matter physics and quantum information processing [1 4]. The lattice is generated by the atom-photon interaction and the setup can be one-, two-, or threedimensional. While primitive Bravais lattices are constructed in most experiments with a single site per unit cell [5, 6], there has been a growing theoretical and experimental effort to build non-standard optical lattices with a few-site basis called superlattices and study the quantum information processing therein [7,9]. One- and two-qubit gates have been realized in a standard two-dimensional (2D) superlattice [10-12]. The potential application is so diverse that a search for more exotic superlattices and a higher degree of tunability in the lattice parameters have been of significant interest.

In contrast to the coupling strengths in conventional 2D superlattices, those of two neighboring particles are the same along all directions and the isolated structures have different interplaquette coupling strengths; therefore, we can study the interaction between two singlets [12] in isolated square superlattices. Furthermore, since the triangular and hexagonal superlattices are framed by three laser beams, there are three equivalent directions in such structures [13 17]. Therefore, we can find the entanglement of three particles or three dimers, which is similar to the Efimov state [18].

In this paper, we propose several schemes to realize isolated structures in a $2 \mathrm{D}$ optical superlattice. The lattice potentials are calculated using the $E^{[2+\epsilon]} \operatorname{method}$ [19, 20], which takes atom-photon interactions as well as hyperfine interactions into account and is therefore appropriate for computing the light shift of hyperfine energy levels. By overlapping the maxima of lattices, the isolated structures are created, while the interference of minima can generate various "sublattice" patterns. We can also control the superlattice parameters such as symmetries and barrier height of "sublattices" by simply tuning the relative phase of the laser beam. In a 2D optical lattice, structures such as hexagonal, triangular, and square have been realized experimentally; hence, we overlay two such structures with different periods and construct the isolated hexagonal lattice, isolated triangular lattice, and isolated square lattice by using the superlattice techniques.

The remainder of this manuscript is organized as follows. In Sec. II, we briefly introduce the $E^{[2+\epsilon]}$ method for calculating the ac Stark shift of hyperfine levels of alkali-metal atoms. 
In Sec. III, we show how to realize various isolated lattice structures and how the geometry of the sublattice can be tuned dynamically. In Sec. IV, we discuss the potential application of our configurations.

\section{THE $E^{[2+\epsilon]}$ METHOD FOR OPTICAL LATTICE POTENTIALS}

An optical lattice is a light-induced periodic potential in space. In order to calculate the potential "felt" by an atom in its hyperfine states, we have to treat both the atomphoton interaction and hyperfine interaction as perturbations. Here, we briefly introduce the $E^{[2+\epsilon]}$ method, which can be used to calculate the lattice potential for hyperfine levels of alkali-metal atoms.

In the dipole approximation, the Hamiltonian of atom-photon interaction is $H_{e}=\vec{p} \cdot \vec{E}$, where $\vec{p}=-e \vec{r}$ is the electric dipole moment, $\vec{r}$ the position of the electron, $e$ the elementary charge, and $\vec{E}$ the electric field vector. Using the Born-Oppenheimer approximation and the long-wavelength approximation, this expression can be further simplified as

$$
\begin{aligned}
\vec{E}(\vec{R}, t) & =K(\vec{R}) e^{-i \omega_{L} t}+K^{\dagger}(\vec{R}) e^{-i \omega_{L} t} \\
K(\vec{R}) & =\sum_{i} \hat{\epsilon}_{i} e^{i \overrightarrow{k_{i}} \cdot \vec{R}}
\end{aligned}
$$

where $\vec{R}$ is the position of the nuclei. Here, we only consider the far-off-resonance laser fields with the same angular frequency $\omega_{L} / 2$ but with different wave vectors $\overrightarrow{k_{i}}$ and polarizations $\hat{\epsilon}_{i}$. The total light shift of an atom is simply the sum of light shifts induced by laser fields with different frequencies.

We now calculate the lattice potential for the energy level $|i\rangle=\left|n_{i} I J_{i} F_{i} M_{F_{i}}\right\rangle$, where $n$ is the principal quantum number, $I$ is the nuclear spin, $J$ is the electronic total angular momentum, and $F=I+J$ is the total angular momentum. $M_{I}, M_{J}$, and $M_{F}$ are the projections of $I, J$, and $F$ on the $z$ axis, respectively, where the $z$ axis is taken as the quantization axis. For hyperfine structures, both the hyperfine interaction and the atomphoton interaction should be treated as perturbations, and the optical lattice potential $U_{i}(\vec{R})$ 
a) $m_{F}=0$
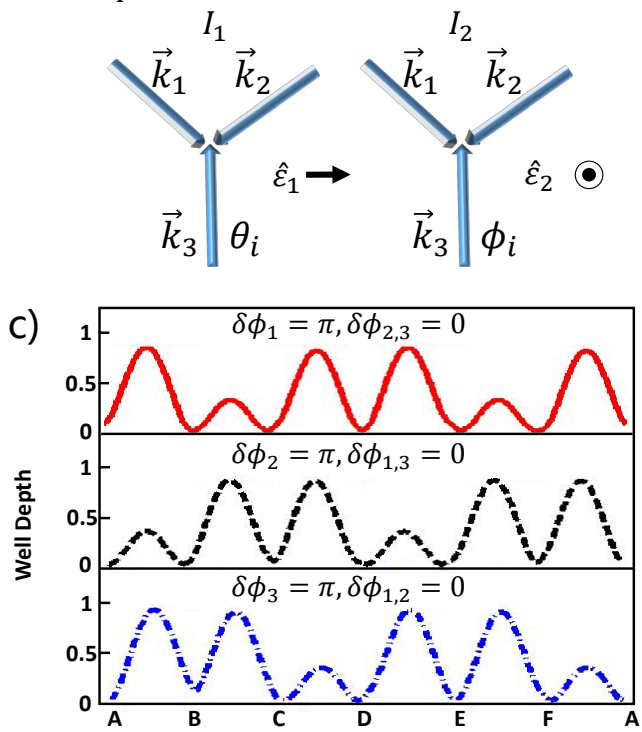

b)

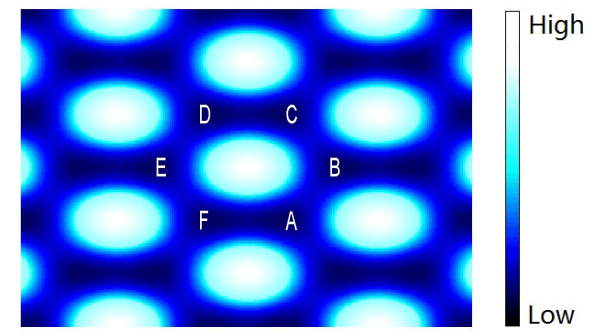

d)

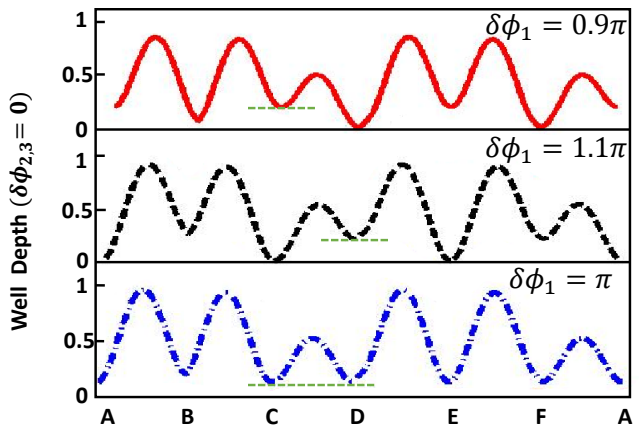

FIG. 1. (Color online) The double well configuration in a hexagonal lattice. (a) The setup for $m_{F}=0$ state. The lattice is formed by two sets of three-laser-beam systems with in-plane and out-of-plane polarizations. (b) The 2D optical potential for a left-right double well configuration is shown with a scaled colormap. A unit cell contains six sites labeled $A, B, C, D, E$ and $F$. The double well is formed between two potential minimums such as $F-A$ and $D-C$. (c) Optical potential along the channel $A-B-\ldots-F-A$. Adjusting the relative phases and making one $\delta \phi_{i}=\theta_{i}-\phi_{i}=\pi, i=1,2,3$, meanwhile keeping the other two $\delta \phi_{i}=0$ allows a site pairing with all three nearest neighbors, therefore the left-right (blue dot-dashed), lower-left (black dashed) and lower-right (red solid) double well configurations can be created, respectively. A double well is formed by two sites with a lower barrier between them. (d) Optical potential along the channel $A-B-\ldots-F-A$. Small deviation of $\delta \phi_{i}$ from $\pi$ can break the double well symmetry and the relative offset between adjacent sites can be tuned. A demonstration using left-right double well configuration is shown where the symmetry in $F-A$ or $D-C$ is broken. The value of $\delta \phi_{1}$ deviate from $\pi$ while $\delta \phi_{2,3}=0$. 
is

$$
\begin{aligned}
& U_{i}(\vec{R})= \\
& -3 \pi c^{2} I_{L} \sum_{j \neq i} \frac{A_{\mathrm{J}_{\mathrm{ji}}}\left(2 F_{j}+1\right)\left(2 F_{i}+1\right)\left(2 J_{j}+1\right) \omega_{\mathrm{F}_{\mathrm{ji}}}}{\omega_{\mathrm{J}_{\mathrm{ji}}}^{3}\left(\omega_{\mathrm{F}_{\mathrm{ji}}}^{2}-\omega^{2}\right)} \\
& \times\left\{\begin{array}{ccc}
J_{i} & J_{j} & 1 \\
F_{j} & F_{i} & I
\end{array}\right\} \sum_{p=0, \pm 1}^{2}\left(\begin{array}{ccc}
F_{j} & 1 & F_{i} \\
M_{\mathrm{F}_{\mathrm{j}}} & p & -m_{\mathrm{F}_{\mathrm{i}}}
\end{array}\right)^{2}\left|K_{p}(\vec{R})\right|^{2},
\end{aligned}
$$

where $I_{L}=(\hbar N \omega) /\left(\epsilon_{0} V\right)$ is the light intensity, $A_{\mathrm{J}_{\mathrm{ji}}}$ is the Einstein coefficient for the finestructure transition between $|i\rangle$ and $|j\rangle, \hbar \omega_{J_{j i}}$ is the difference between fine-structure energies, and $\hbar \omega_{F_{j i}}$ is $\hbar \omega_{J_{j i}}$ plus the difference between hyperfine energy corrections. The term inside curly brackets is a $6 J$ symbol, while that inside the large round brackets is the $3 J$ symbol, which describes the selection rules and relative strength of the transitions. The only

term concerning the light fields is $K_{p}(\vec{R})$, which is the pth $(p=0, \pm 1)$ spherical component of the rank-1 tensor $K(\vec{R})$. From another perspective, it also stands for the different polarization components of the laser fields $(p=1,0,-1$ for right-handed, linear, and left-handed polarization, respectively). As we can see, $E^{[2+\epsilon]}$ has a very compact form and can easily incorporate experimental atomic structure data; therefore, it is useful for the lattice-potential calculations of many elements.

Here, we take the hyperfine ground states $\left|F=1, m_{F}=0, \pm 1\right\rangle$ of ${ }^{87} R b$ as an example and further simplify Eq. (2). Because $5 s^{2} S_{1 / 2}-5 p^{2} P_{1 / 2}$ and $5 s^{2} S_{1 / 2}-5 p^{2} P_{3 / 2}$ are the two primary transition lines with a much larger Einstein coefficient $A_{J}$ and much smaller transition energy $\omega_{J}$, we can neglect other excited energy levels and only consider these two lines as a good approximation. In the following sections, a beam is regarded as red or blue detuned only when it is red or blue detuned to both the transition lines. The formula Eq. (2) includes the scalar, vector, and tensor light shifts.

\section{ADJUSTING SUPERLATTICE PARAMETERS FOR NOVEL LATTICE GEOME- TRIES}

To construct the isolated structures, we need to not only overlay commensurate lattices with different periodicity, but also tune their relative positions and depth, which requires a high degree of tunability in experimental setups. One advantage of the optical lattice is that the lattice parameters can be controlled by adjusting the intensity, polarization, and 
relative phase of the laser beam, and an even richer variety of lattice structures can be constructed and controlled if we operate in superlattices. Here, we take double wells in a 2D hexagonal lattice as an example and demonstrate the new lattice geometries created in these superlattices.

A hexagonal optical lattice can be constructed using three laser beams with equal intensity and equal frequency that intersect in the xy plane with mutually enclosing angles of $2 \pi / 3$ [6]. The lattice potential is modulated for different Zeeman levels [21]. As shown in Fig. (17), the double-well configuration can be realized for the $m_{F}=0$ state by using two sets of

three-laser-beam systems: one with wave vectors $\overrightarrow{k_{i}}$ and in-plane polarizations $\hat{\epsilon_{1}}$, and the other with the same wave vectors but out-of-plane polarizations $\hat{\epsilon_{2}}$. The in-plane polarized beams have intensity $I_{1}$ and phases $\theta_{i}$, while the out-of-plane polarized beams have intensity $I_{2}$ and phases $\phi_{i}$. Each set will generate a hexagonal lattice, and changing the phase of a certain beam will cause the lattice to shift in the direction of the corresponding wave vector.

Since there is no interference for $m_{F}=0$, by changing the relative phase $\delta \phi_{i}=\theta_{i}-\phi_{i}$ between corresponding laser beams of two lattices, we can shift the in-plane lattice and place its minima between any two out-of-plane lattice minima; consequently, a double-well configuration can be created, as shown in Fig. (11b), where we demonstrate a left-right double-well configuration. The labels $A-F$ are the six sites in one unit cell. In Fig. (11), the optical potentials inside one hexagonal unit cell for the left-right, lower-left, as well as lower-right double-well configurations are calculated, and the phase setups are presented, where the double well is formed by two potential minimums with a lower barrier between them.

The tilt of the double well can be controlled as well through an adjustment of the relative phases. For example, in the left-right double well, we can adjust $\delta \phi_{1}$ while keeping $\delta \phi_{2,3}=0$, as shown in Fig. (1) ). The barrier height of the double well can also be adjusted by changing the relative laser intensity of in-plane and out-of-plane laser beams $I_{1} / I_{2}$.

\section{ISOLATED STRUCTURES IN SUPERLATTICE}

The construction of isolated structures in 2D requires an overlapping of multiple commensurate lattices with different periodicities. In general, the barrier between isolated structures is created by overlaying the maxima of lattices, while the structure inside one "sublattice" is 
a)

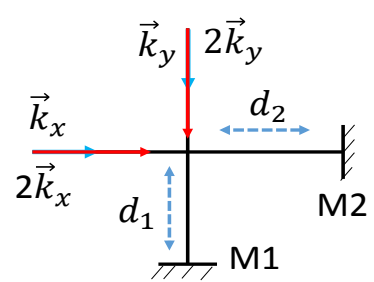

c)

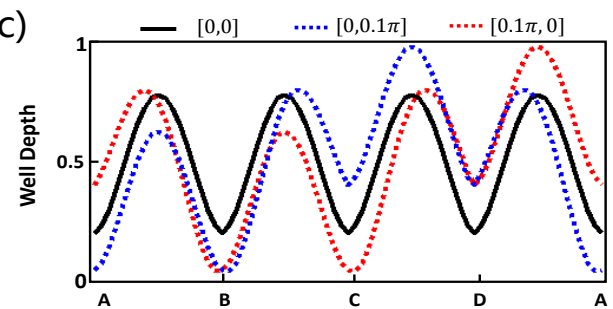

b)

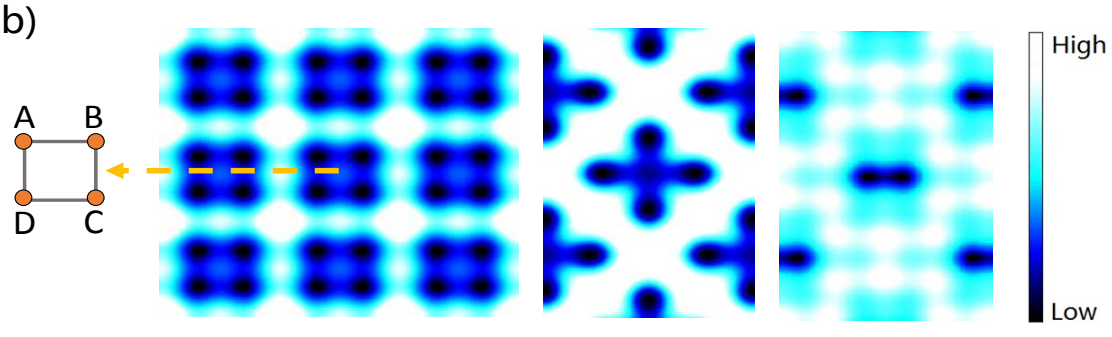

FIG. 2. (Color online) The isolated square superlattice. (a) The setup includes two standing waves for each frequency. Different polarizations and detunings can generate different geometries, such as (b) the isolated square (left), the isolated crosses (middle) and the isolated double wells (right). The isolated square configuration using in-plane polarizations. $\vec{k}_{i}$ lasers are red detuned, meanwhile $2 \vec{k}_{i}$ lasers are blue detuned, with $i=x, y$. A unit cell of the superlattice contains four sites $A, B, C$ and $D$. (c) Optical potential along the channel $A-B-C-D$ in the isolated square. Adjustment of the relative phases $\left[\theta_{1}-\phi_{1}, \theta_{2}-\phi_{2}\right]$ changes the relative depth between different sites. The $\mathrm{x}$-axis symmetry (red dashed) or the y-axis symmetry (blue dashed) can be broken using different phase setups. The black solid line is the optical potential for a symmetric square sublattice.

determined by the interference pattern of the minima. In the experiment, the tuning of the relative position between lattices can be realized by changing the relative phases between laser beams, as demonstrated in the previous section. Because hexagonal, triangular, and square structures have been realized in a 2D Bravais lattice, overlaying two such lattices with different periodicities can generate the isolated hexagonal lattice, isolated triangular lattice, and isolated square lattice, respectively. 


\section{Isolated Square Superlattice}

The 2D square superlattice can be constructed using eight beams in four perpendicular directions and with two frequencies $\omega_{L}$ and $2 \omega_{L}$. Here, we recommend the setup introduced in Fig. (2a) for each frequency, where the lattice is formed by two standing waves reflected by mirrors $M 1$ or $M 2$. For the $x$ direction, the wave vectors of incoming beams are $\vec{k}_{x}$ and $2 \vec{k}_{x}$, and the effective distance is $d_{1}$ before the beam returns to the atomic cloud. For the

$y$ direction, the wave vectors are $\vec{k}_{y}$ and $2 \vec{k}_{y}$ and the effective distance is $d_{2}$, where $\left|\vec{k}_{y}\right|=$ $\left|\vec{k}_{x}\right|=k=\omega_{L} / c$, with $c$ representing the speed of light in vacuum. The retro-reflected beams provide intrinsic topological phase stability while providing sufficient freedom for parameter adjustments. Because of the absence of the cross terms, the total light shifts equal the sum of the light shifts induced by laser fields with different frequencies. Therefore, by changing the relative position and light intensity, we can generate different lattice configurations such as isolated squares, isolated double wells, or isolated crosses, as demonstrated in Fig. (2p).

Here, we focus on the isolated square lattice for the $m_{F}=0$ atoms. We can use either in-plane or out-of-plane lattices to generate this structure. We take an in-plane lattice as an example, where the red-detuned $\omega_{L}$ beams $\vec{E}_{1}$ and the blue-detuned $2 \omega_{L}$ beams $\vec{E}_{2}$ are both polarized in the $x y$ plane. The spatial dependence of the electric field is

$$
\begin{aligned}
\vec{E}_{1} & \propto\left(e^{i\left(k x+\theta_{1}\right)}+e^{i\left(-k x+\theta_{1}+2 k d_{1}\right)}\right) \hat{y} \\
& +\left(e^{i\left(k y+\theta_{2}+2 k d_{2}\right)}+e^{i\left(-k y+\theta_{2}\right)}\right) \hat{x} \\
\vec{E}_{2} & \propto\left(e^{i\left(2 k x+\phi_{1}\right)}+e^{i\left(-2 k x+\phi_{1}+4 k d_{1}\right)}\right) \hat{y} \\
& +\left(e^{i\left(2 k y+\phi_{2}+4 k d_{2}\right)}+e^{i\left(-2 k y+\phi_{2}\right)}\right) \hat{x}
\end{aligned}
$$

When $\theta_{1}=\phi_{1}, \theta_{2}=\phi_{2}$, a symmetric sublattice structure of isolated squares is formed, as shown in Fig. (2b), where the four sites in one unit cell of the superlattice are labeled $A, B, C$, and $D$. Notice that the change of $d_{1}$ and $d_{2}$ does not change the geometry but only shifts the lattice as a whole. The height of the barrier is mostly determined by the intensity of basis-frequency laser beams, and the depth of the square lattice wells is primarily affected by the intensity of double-frequency laser beams. The symmetry of the isolated square can be controlled by shifting the relative position of the two lattices. For instance, we can break the y-axis symmetry by setting $\theta_{1}=\phi_{1}, \theta_{2}-\phi_{2}=0.2 \pi$, which makes the site depths $U_{A}=$ $U_{B} \neq U_{C}=U_{D}$, or we can break x-axis symmetry by setting $\theta_{1}-\phi_{1}=0.2 \pi, \theta_{2}=\phi_{2}$, which results in $U_{A}=U_{D} \neq U_{C}=U_{B}$. The optical potential along the channel $A-B-C-D-A$ 

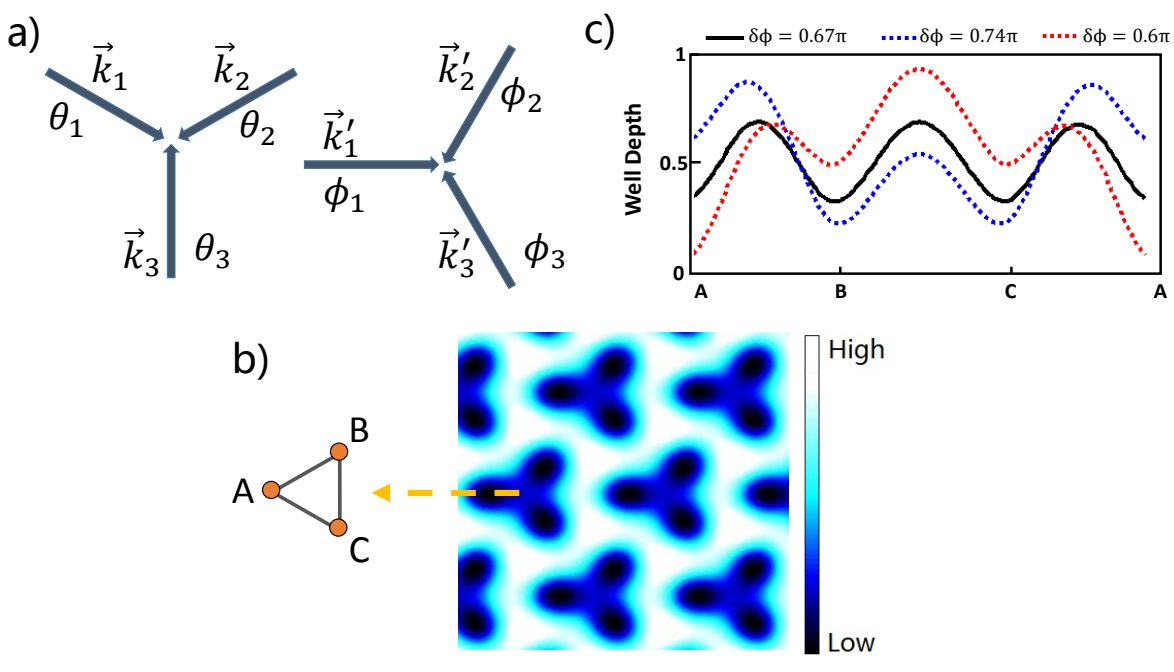

FIG. 3. (Color online) The isolated triangular superlattice. (a) The setup for $m_{F}=0, \pm 1$ states. The lattice is formed by two sets of three-laser-beam systems. The detuning and polarization depend on the $m_{F}$ state trapped. (b) The 2D optical potential for the isolated triangular configuration. A unit cell of the superlattice contains three sites $A, B$ and $C$. (c) Optical potential along the channel $A-B-C-A$. By altering $\delta \phi=\theta_{1}-\phi_{1}$, we can make $A$ site depth larger (blue dashed), smaller (red dashed) or equal (black solid) compared to $B$ and $C$ site.

of the symmetric, $\mathrm{x}$-axis nonsymmetric, and $\mathrm{y}$-axis nonsymmetric structures is shown in Fig. (2c).

\section{Isolated Triangular Superlattice}

The setup requires two sets of three-laser-beam systems: one set with wave vectors $\overrightarrow{k_{i}}$ $(i=1,2,3)$, frequency $\omega_{L}$, and phases $\theta_{i}$, and the other one with $\overrightarrow{k_{i}^{\prime}}$, frequency $\omega_{L}^{\prime}=\sqrt{3} \omega_{L}$, and phases $\phi_{i}$, as shown in Fig. (3a). For the $m_{F}= \pm 1$ state, each set of beams can be either in-plane or out-of-plane polarized, but both need to be red detuned. For $m_{F}=0$ states, each set can be either out-of-plane polarized and red detuned or in-plane polarized and blue detuned.

The $\omega_{L}$ and $\omega_{L}^{\prime}$ lasers both generate a triangular lattice. By changing the relative phases of laser beams and placing the minimum of the $\omega_{L}$ lattice at the center of the three minima of the $\omega_{L}^{\prime}$ lattice, we can create the isolated triangular lattice structure, as shown in Fig. 
(3b), where the three sites in one unit cell of the superlattice are labeled by $A, B$, and $C$. The barrier height inside one triangular "sublattice" largely depends on the intensity of the $\omega_{L}^{\prime}$ beams, while the outer barrier between two triangular "sublattices" depends on the intensity of $\omega_{L}$ beams.

In Fig. (3c), we calculate the 2D optical potential along the channel $A-B-C-A$, and the lattice potential of a symmetric isolated triangular is shown with a blue dot-dashed line, where the sites $A, B$, and $C$ have the same depth and same barrier height. By shifting the $\omega_{L}$ lattice away from the center, we can break the triangular symmetry and adjust the relative depth of three minima at will. For example, by altering $\delta \phi=\theta_{1}-\phi_{1}$, we can horizontally shift the $\omega_{L}$ lattice and make the depth of site $A$ larger (blue dashed) or smaller (red dashed) than those of sites $B$ and $C$, which is demonstrated in Fig. (3r). Similarly, a change of $\theta_{2}-\phi_{2}$ or $\theta_{3}-\phi_{3}$ can make the depth of site $B$ or $C$ different from those of the other two.

\section{Isolated Hexagonal Superlattice}

We can use a similar setup to form an optical lattice with isolated hexagonal structures. The setup requires two sets of three-laser-beam systems: one set with wave vector $\overrightarrow{k_{i}}$ $(i=1,2,3)$ and frequency $\omega_{L}$, which is red detuned, and the other one with $\overrightarrow{k_{i}^{\prime}}$ and frequency $\omega_{L}^{\prime}=\sqrt{3} \omega_{L}$, which is taken to be blue detuned. The two sets are perpendicular to each other, as shown in Fig. (4a). For the $m_{F}=0$ state, all $\omega_{L}$ and $\omega_{L}^{\prime}$ laser beams need to be out-of-plane polarized, while for $m_{F}= \pm 1$, each set can be either in-plane or out-of-plane polarized. The $\omega_{L}$ and $\omega_{L}^{\prime}$ lasers generate a triangular and hexagonal lattice, respectively. By changing the relative phase between the two sets of laser beams and overlapping the maximum of the $\omega_{L}^{\prime}$ lattice with that of the minima of the $\omega_{L}$ lattice, we can create a $2 \mathrm{D}$ lattice with isolated hexagonal structures, as shown in Fig. (4b).

The essence of creating the isolated structures is to build a high barrier between "sublattices," which could be a result of increasing the depth of the "bigger" lattice or a result of overlapping the maxima of different lattices. The pattern inside each "sublattice," however, is a superposition of minima; therefore, a change of relative depth and position can greatly 
a)

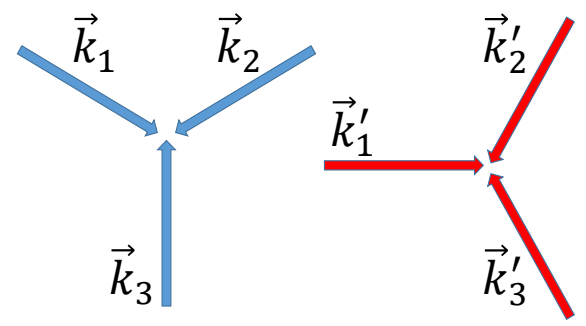

b)

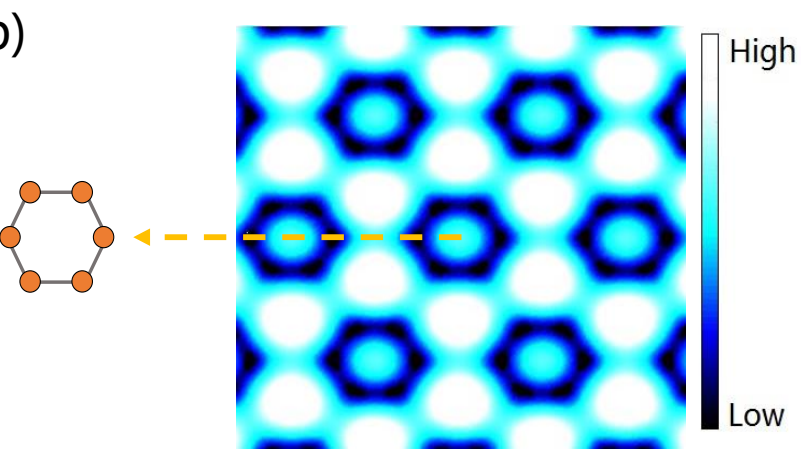

FIG. 4. (Color online) The isolated hexagonal superlattice. (a) The setup for $m_{F}=0, \pm 1$ states. The lattice is formed by two sets of three-laser-beam systems, one red detuned and the other blue detuned. (b) The 2D optical potential for the isolated hexagonal configuration with a scaled colormap.

influence its shape as well as symmetry. In theory and experiments, the pattern inside each "sublattice" is usually more interesting and important than the pattern of "sublattices." However, only three patterns are introduced in this section because these three patterns are very basic and representative. Many other structures that are more complex can be created in a similar manner.

\section{DISCUSSIONS AND CONCLUSIONS}

We demonstrated the lattice potential for the ground hyperfine state of ${ }^{87} R b$ by using the $E^{[2+\epsilon]}$ method to calculate the light shift of hyperfine energy levels and, thereby, lattice potential. In fact, since the hyperfine corrections are very small, conventional second-order perturbation theory $E^{[2]}$ applied to hyperfine structures serves the purpose, and we can replace $\omega_{\mathrm{F}_{\mathrm{ji}}}$ in Eq. (2) with $\omega_{\mathrm{J}_{\mathrm{ji}}}$. However, when we are concerned with the different lattice potentials "felt" by two hyperfine energy states of the ground state, only $E^{[2+\epsilon]}$ can provide the difference, while $E^{[2]}$ provides identical results for the two states owing to the absence of hyperfine interactions. Such a situation may arise when we study the dynamics of a spinor Bose-Einstein condensate (BEC) trapped in an optical lattice.

The isolated lattices are good candidates for studying multiple-particle entanglement and few-body physics. Once the barrier height exceeds the chemical potential, the atoms are 
trapped in one isolated sublattice, which can be seen as an isolated subsystem, and its static as well as the dynamic properties can be measured. On one hand, we can adjust the symmetry of the sublattice and create different degrees of entanglement between different pairs of atoms. On the other hand, by loading a BEC into the 2D isolated "sublattices," we can study the dynamics of this system of coupled bosonic wells, the pairs and triplets of coupled wells of which have been studied extensively in the literature [22, [23]. This isolated structure provides unique opportunities to observe many novel phenomena such as self-trapping, the macroscopic effects of population inversion, and the Efimov state.

In conclusion, compared with the 1D double-well superlattice studied previously, we proposed new schemes to realize isolated structures in 2D optical superlattices by overlapping the maxima of commensurate optical lattices, and various "sublattice" patterns are generated by the interference of minima. By using three different kinds of primitive lattices, we demonstrated the construction of isolated hexagonal, triangular, and square superlattices. These configurations not only generate non-standard lattice geometry, but also provide convenient access for dynamically tuning the lattice parameters, especially to regulate the symmetry of sublattices by adjusting the relative phase. We believe they give an opportunity to observe and study novel physics, and in the future, the lattices presented here can be used for applications in quantum simulation and quantum information processing, especially for constructing new quantum logic gates.

[1] I. M. Georgescu, S. Ashhab, and F. Nori, Quantum simulation, Rev. Mod. Phys 86, 153 (2004)

[2] T. Calarco, U. Dorner, P. S. Julienne, C. J. Williams, and P. Zoller, Quantum computations with atoms in optical lattices: Marker qubits and molecular interactions, Phys. Rev. A 70, 012306 (2004)

[3] L. Niu, D. Hu, S. Jin, X. Dong, X. Chen, X. Zhou, Excitation of atoms in an optical lattice driven by polychromatic amplitude modulation, Opt. Express 23, 10064 (2015)

[4] D. Hu, L. Niu, B. Yang, X. Chen, B. Wu, H. Xiong, and X. Zhou, Long-time nonlinear dynamical evolution for P-band ultracold atoms in an optical lattice, Phys. Rev. A 92043614 $(2015)$

[5] M. Greiner, O. Mandel, T. Esslinger, T. W. Hansch, and I. Bloch, Quantum phase transition 
from a superfluid to a Mott insulator in a gas of ultracold atoms, Nature 41539 (2002)

[6] C. Becker, P. Soltan-Panahi, J. Kronjäger, S. Dörscher, K. Bongs and K. Sengstock, Ultracold quantum gases in triangular optical lattices, New J. Phys. 12065025 (2010)

[7] J. Sebby-Strabley, M. Anderlini, P. S. Jessen, and J. V. Portol, Lattice of double wells for manipulating pairs of cold atoms, Phys. Rev. A 73033605 (2006)

[8] L. Santos, M. A. Baranov, J. I. Cirac, H. U. Everts, H. Fehrmann, and M. Lewenstein, Atomic quantum gases in Kagomé lattices, Phys. Rev. Lett. 93030601 (2004)

[9] G. B. Jo, J. Guzman, C. K. Thomas, P. Hosur1, A. Vishwanath, and D. M. Stamper-Kurn, Ultracold atoms in a tunable optical kagome lattice, Phys. Rev. Lett. 108045305 (2012)

[10] J. K. Pachos and P. L. Knight, Quantum computation with a one-dimensional optical lattice, Phys. Rev. Lett. 91108103 (2003)

[11] G. K. Brennen, C. M. Caves, P. S. Jessen, and I. H. Deutsch, Quantum logic gates in optical lattices, Phys. Rev. Lett. 821060 (1999)

[12] L. Jiang, A. M. Rey, O. Romero-Isart, J. J. Garca-Ripoll, A. Sanpera, and M. D. Lukin, Preparation of decoherence-free cluster states with optical superlattices, Phys. Rev. A 79.2 022309 (2009)

[13] K. Nemoto, C. A. Holmes, G. J. Milburn, and W. J. Munro, Quantum dynamics of three coupled atomic Bose-Einstein condensates, Phys. Rev. A 63013604 (2000)

[14] M. Hiller, T. Kottos, and T. Geisel, Complexity in parametric Bose-Hubbard Hamiltonians and structural analysis of eigenstates, Phys. Rev. A 73 061604(R) (2006)

[15] A. R. Kolovsky, Semiclassical quantization of the Bogoliubov spectrum, Phys. Rev. Lett. 99 $020401(2007)$

[16] R. Franzosi and V. Penna, Self-trapping mechanisms in the dynamics of three coupled BoseEinstein condensates, Phys. Rev. A 65013601 (2001)

[17] P. Hsieh, C. Chung, J. McMillan, M. Tsai, M. Lu, N. Panoiu, and C. W. Wong, Photon transport enhanced by transverse Anderson localization in disordered superlattices, Nat. Phys. 11268 (2015)

[18] M. Kunitski, S. Zeller, J. Voigtsberger, A. Kalinin, L. P. H. Schmidt, M. Schöffler, A. Czasch, W. Schöllkopf, R. E. Grisenti, T. Jahnke, et al., Observation of the Efimov state of the helium trimer, Science $\mathbf{3 4 8} 551$ (2015)

[19] X. Zhou, X. Xu, X. Chen, J. Chen, Magic wavelengths for terahertz clock transitions, Phys. 
Rev. A 81012115 (2010)

[20] X. Xu, B. Qing, X. Z. Chen, X. J. Zhou, A simplified method for calculating the ac Stark shift of hyperfine levels of alkali-metal atoms, Phys. Lett. A 3791347 (2015) 
[21] P. Soltan-Panahi, J. Struck, P. Hauke, A. Bick, W. Plenkers, G. Meineke, C. Becker, P. Windpassinger, M. Lewenstein and K. Sengstock, Multi-component quantum gases in spindependent hexagonal lattices, Nat. Phys. 7434 (2011)

[22] A. S. Parkins and D. F. Walls, The physics of trapped dilute-gas BoseEinstein condensates, Phys. Rep. 3031 (1998)

[23] F. Dalfovo, S. Giorgini, L. P. Pitaevskii, and S. Stringari, Theory of Bose-Einstein condensation in trapped gases, Rev. Mod. Phys. 71463 (1999) 